\title{
Electrical band flattening, valley flux, and superconductivity in twisted trilayer graphene
}

\author{
Alejandro Lopez-Bezanilla ${ }^{1}$ and J. L. Lado $\odot^{2}$ \\ ${ }^{1}$ Theoretical Division, Los Alamos National Laboratory, Los Alamos, New Mexico 87545, USA \\ ${ }^{2}$ Department of Applied Physics, Aalto University, 00076 Aalto, Espoo, Finland
}

(Received 10 May 2020; accepted 18 August 2020; published 3 September 2020)

\begin{abstract}
Twisted graphene multilayers have been demonstrated to yield a versatile playground to engineer controllable electronic states. Here, by combining first-principles calculations and low-energy models, we demonstrate that twisted graphene trilayers provide a tunable system where Van Hove singularities can be controlled electrically. In particular, it is shown that besides the band flattening, bulk valley currents appear, which can be quenched by local chemical dopants. We finally show that in the presence of electronic interactions, a nonuniform superfluid density emerges whose nonuniformity gives rise to spectroscopic signatures in dispersive higher-energy bands. Our results put forward twisted trilayers as a tunable van der Waals heterostructure displaying electrically controllable flat bands and bulk valley currents.
\end{abstract}

DOI: 10.1103/PhysRevResearch.2.033357

\section{INTRODUCTION}

The interplay between topology and correlations represents a highly fruitful area in condensed matter physics. However, exploring unconventional states of matter requires identifying systems where electronic correlations, topology, and electronic dispersions can be realistically controlled. In this regard, twisted van der Waals materials [1-8] provide a powerful solid state platform to realize exotic quantum phenomena. The tunability of twisted van der Waals materials stems from the emergence of a band structure that can be controlled by the twist between different two-dimensional materials $[9,10]$. In particular, the different quantum states in twisted graphene systems stem from the possibility of controlling the ratio between kinetic and interaction terms. In twisted graphene bilayers, such tunability has allowed the realization of superconductivity $[1,2,4]$, correlated insulators, topological networks [5,6], Chern insulators [11], and quasicrystals [12-15]. As a result, current experimental efforts are focusing on exploring new twisted van der Waals materials, with the aim of finding platforms that allow for an even higher degree of control [16,17].

From a quantum engineering point of view, applying a perpendicular bias between layers [18] provides a versatile way of tuning correlated states in twisted graphene multilayers. This has been demonstrated in paradigmatic examples of correlated states in twisted tetralayers (double bilayers) $[19,20]$ and twisted trilayers (monolayers/bilayers) $[16,17]$. Moreover, interlayer bias is known to generate internal valley currents in twisted graphene bilayers [5,6,21,22], creating topological networks at low angles [5,6,21] and generating valley fluxes in flat band regimes [22]. This interplay of correlations and topology in twisted graphene multilayers makes

Published by the American Physical Society under the terms of the Creative Commons Attribution 4.0 International license. Further distribution of this work must maintain attribution to the author(s) and the published article's title, journal citation, and DOI. these materials a powerful platform to explore exotic states of matter [23-26] in a realistically feasible manner.

From a theoretical point of view, electronic structure calculations of a twisted graphene bilayer conducted with real-space tight-binding models [10] or continuum Dirac descriptions [9] capture the fundamental features of the electronic dispersion. Nevertheless, internal coordinate optimization can quantitatively modify the electronic dispersion [27-34]. Well known examples of this are the growth of $\mathrm{AB} / \mathrm{BA}$ regions in twisted bilayers [29,35]. It is important to note that studying twisted graphene multilayers from first principles represents a remarkable challenge, due to the large number of atoms present in a unit cell.

Here, by combining first-principles calculations and lowenergy models, we show that twisted graphene trilayers [36] host flat bands whose bandwidth can be controlled electrically. We address the impact of an interlayer bias from both first-principles and effective models, showing that Van Hove singularities can be merged electrically. We show that associated with the interlayer bias, bulk valley currents emerge, which are impacted by the existence of chemical impurities in the system. We finally address the superconducting states in these doped trilayers, showing that the nonuniform superfluid density has an impact in high-energy bands.

Our paper is organized as follows. In Sec. II we show the electronic structure of a twisted graphene trilayer from both first-principles and low-energy models. In Sec. III we explore in detail the impact of an electric field. In Sec. IV we explore the effect of chemical impurities. In Sec. V we address the impact of an emergent nonuniform superfluid density. In Sec. VI we summarize our conclusions.

\section{ELECTRONIC STRUCTURE OF TWISTED TRILAYER GRAPHENE}

The electronic structure of twisted graphene trilayers [36-41] shows different features in comparison with twisted graphene bilayers [10,42]. The electronic structure of small 

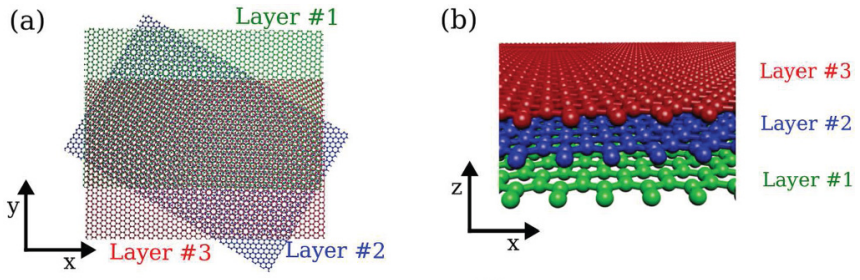

(c)

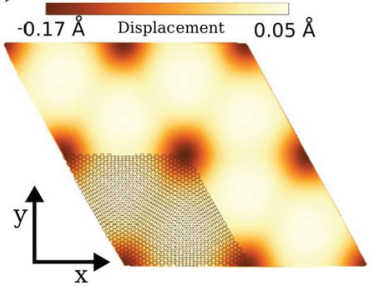

(d)

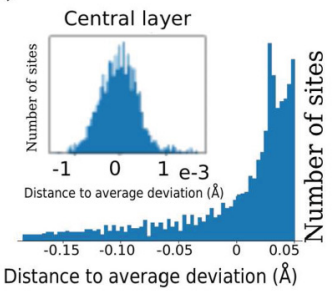

FIG. 1. (a) and (b) Sketch of the structure of a twisted graphene trilayer, in which the top and bottom layers are aligned and are twisted with respect to the middle one. (c) Colormap of a DFT-based relaxed $2 \times 2$ supercell of a twisted trilayer. The colored pattern corresponds to the difference $\Delta=z-z_{a v}$ between the $z$ coordinate of an atom in the surface layer and the average coordinate $z_{a v}$ of all atoms in the same layer. The AA stacking regions are colored with darker tones whereas $\mathrm{AB} / \mathrm{BA}$ stacking regions are colored with lighter tones. Superimposed is the unit cell of the trilayer. (d) Histogram showing the number of occurrences of each distance to the average deviation value. Tiny deviations from flatness occur in the central layer. Calculations were performed with DFT.

magic angle twisted bilayer graphene features four flat bands lying around the Fermi level, with band splitting at the $\Gamma$ point of the Brillouin zone of the emergent moiré superlattice $[10,42]$. Density functional theory (DFT) calculations have shown results consistent with those effective models in twisted graphene multilayers, yet quantitative modifications are observed when including relaxation of the atomic coordinates [29,30,43-45] and crystal-field effects [34,46]. Therefore, to benchmark the electronic properties of twisted graphene multilayers, it is essential to start from a correct description that takes into account the geometric corrugation and $a b$ initio electrostatics of the moiré system.

We consider a twisted trilayer structure in which the upper and lower layers are aligned and the middle one is twisted with an angle $\theta$ with respect to those [Figs. 1(a) and 1(b)]. In particular, in the following we consider a twisted trilayer whose middle layer has a twisting angle of $1.9^{\circ}$ with respect to the external layers. A twisted multilayer like this can be created with standard tear, rotate, and stack techniques [47]. The system of 5514 atoms is fully relaxed, allowing for lateral and vertical displacement of the $\mathrm{C}$ atoms in the structure. Figure 1(c) shows the colormap of one of the two equivalent external layer relaxations. The color scheme represents the vertical variation of each $\mathrm{C}$ atom at the surface with respect to the average deviation within each layer. The darker areas indicate a displacement of atoms out of the surface which occurs predominantly in the AA stacking region. The histogram of Fig. 1(d) shows that the number of atoms in the upper layer whose vertical coordinate is above the average is twice as

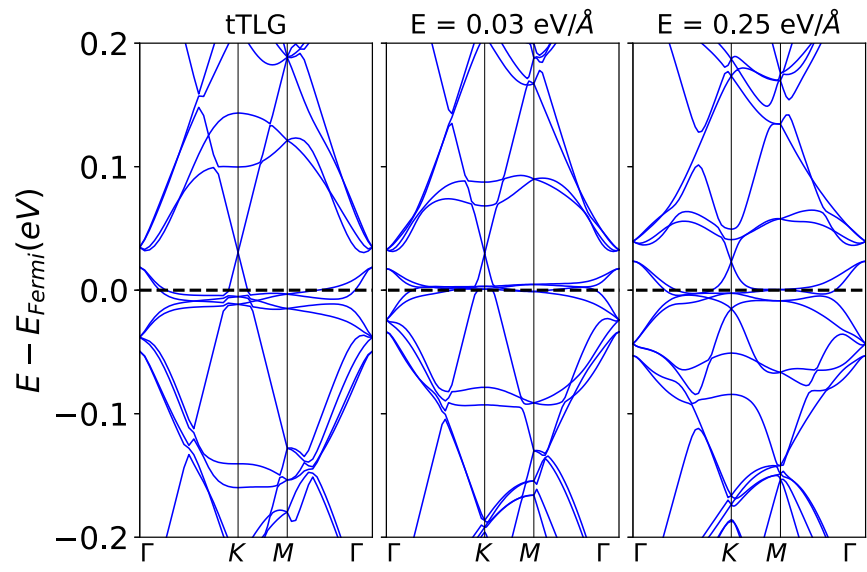

FIG. 2. Electronic band diagrams of twisted trilayer graphene. Localized states at the Fermi energy coexist with highly dispersive state bands forming a Dirac cone in the conduction band. Applying a small electric field perpendicular to the TTG, a reduction of all band dispersions is induced. A stronger electric field removes the Dirac cones and induces hybridization between electronic states. Calculations were performed with DFT.

large as the atoms displaced in the opposite direction below the average.

The first-principles electronic band diagram of the fully relaxed structure of $\theta=1.9^{\circ}$ twisted trilayer graphene (TTG) is shown in Fig. 2. In contrast with twisted bilayer graphene, two highly dispersive bands coexist with four low-dispersive bands grouped at the Fermi energy. The Dirac-like crossing above the charge neutrality point leads to a small charge transfer between the flat and dispersive bands even at half filling. Additional flattening of the localized states can be induced by application of an external electric field perpendicular to the TTG surface. A field of $0.03 \mathrm{eV} / \AA$ reduces the dispersion of all electronic states in the vicinity of the Fermi energy without inducing any interband charge transfer. Increasing the strength up to $0.25 \mathrm{eV} / \AA$, a disruption of the linear bands is observed and a hybridization of the flat bands with a neighboring state increases their dispersion. It is observed that the application of an interlayer bias generates Dirac crossings above and below charge neutrality, as well as a variety of anticrossings (Fig. 2).

The first-principles calculations above show that the electronic structure of twisted trilayer graphene show strong differences from the one of twisted graphene bilayers. In particular, a highly dispersive set of bands coexists with the nearly flat bands at charge neutrality. In order to explore more in detail the physics and twisted trilayers, in the following we exploit a low-energy model. We find that the tight-binding model qualitatively reproduces the important features of the band structure without including relaxations and additional charge-transfer effects. Therefore, for the sake of simplicity, we now use an unrelaxed structure for our tight-binding model calculations. We take a single orbital per carbon atom, yielding a tight-binding Hamiltonian of the form

$$
\mathcal{H}_{0}=-t \sum_{\langle i, j\rangle, s} c_{i, s}^{\dagger} c_{j, s}-\sum_{i, j, s} \bar{t}_{\perp}\left(\mathbf{r}_{i}, \mathbf{r}_{j}\right) c_{i, s}^{\dagger} c_{j, s},
$$


with $\bar{t}_{\perp}\left(\mathbf{r}_{i}, \mathbf{r}_{j}\right)=t_{\perp} \frac{\left(z_{i}-z_{j}\right)^{2}}{\left|\mathbf{r}_{i}-\mathbf{r}_{j}\right|^{2}} e^{-\beta\left(\left|\mathbf{r}_{i}-\mathbf{r}_{j}\right|-d\right)}$, where $d$ is the interlayer distance and $\beta$ controls the decay of the interlayer hopping. As a reference, for twisted graphene multilayers $t \approx$ $3 \mathrm{eV}$ and $t_{\perp} \approx 0.15 t .{ }^{1}$ Similar real-space models were used to study a variety of twisted graphene multilayers [10,36,50,51], providing a simple formalism to study the effect of dopants and impurities [52,53]. However, in contrast to continuum models $[9,42,54]$, measuring of valley-related quantities with a real-space-based formalism is nontrivial.

Twisted graphene multilayers have an approximate symmetry associated with the valley quantum number [9]. Valley physics in both DFT calculations and the tight-binding model are emergent symmetries, in the sense that valley are not easily defined in terms of real-space chemical orbitals. This limitation can be overcome by defining the so-called valley operator $[21,52,55]$ in the tight-binding description. With the valley operator the expectation value of the valley can be computed in a real-space representation $[22,56]$. The details of the valley operator $[21,52,55]$ are given in Appendix B. In the following we take as the starting point the valley operator $\mathcal{V}_{z}$. With the previous operator, we can compute the valley flavor of each eigenstate of the twisted trilayer supercell within the real-space formalism as $\left\langle\mathcal{V}_{z}\right\rangle=\left\langle\Psi\left|\mathcal{V}_{z}\right| \Psi\right\rangle$. It is worth noting that this operator can be easily defined in the tight-binding basis but not in the DFT basis. We finally note that the valley operator $\mathcal{V}_{z}$ in the twisted moire system will show the valley flavor in the original Brillouin zone of graphene, not in the mini Brillouin zone of the twisted system.

With the previous formalism, we now compute the electronic structure of the low-energy model at the same angle $\theta=1.9^{\circ}$ as in the first-principles calculations (Fig. 3). We note that, besides some additional splittings observed in the first-principles calculations (Fig. 2), the band structure obtained with the low-energy model [Figs. 3(a) and 3(b)] gives comparable results. As shown in Fig. 3(a), in the absence of an interlayer bias the system shows nearly flat bands coexisting with highly dispersive states. Whereas the nearly flat bands are degenerate in valleys in the path shown, the dispersive states belong to different valleys in different parts of the Brillouin zone. It is also observed that the dispersive Dirac cones are slightly displaced from charge neutrality, as observed in the first-principles results.

We now move on to consider the effect of the interlayer bias. An interlayer bias can be easily included in the lowenergy tight-binding model by means of

$$
\mathcal{H}_{V}=\frac{V}{2 d} \sum_{i, s} z_{i} c_{i, s}^{\dagger} c_{i, s},
$$

where $z_{i}$ is the $z$ position of site $i, d$ is the interlayer distance, and $V$ is the strength of the interlayer bias. The full Hamiltonian in the presence of interlayer bias is thus $\mathcal{H}=\mathcal{H}_{0}+\mathcal{H}_{V}$. When an interlayer bias is turned on [Fig. 3(b)], the valley degeneracy in the $G-K$ path is lifted and the dispersive bands

\footnotetext{
${ }^{1}$ At low energies the spectrum is invariant upon rescaling of the interlayer coupling, which allows one to explore effective smaller angles with smaller unit cells $[48,49]$. Our calculations are performed with a rescaled $t_{\perp}=0.4 t$.
}
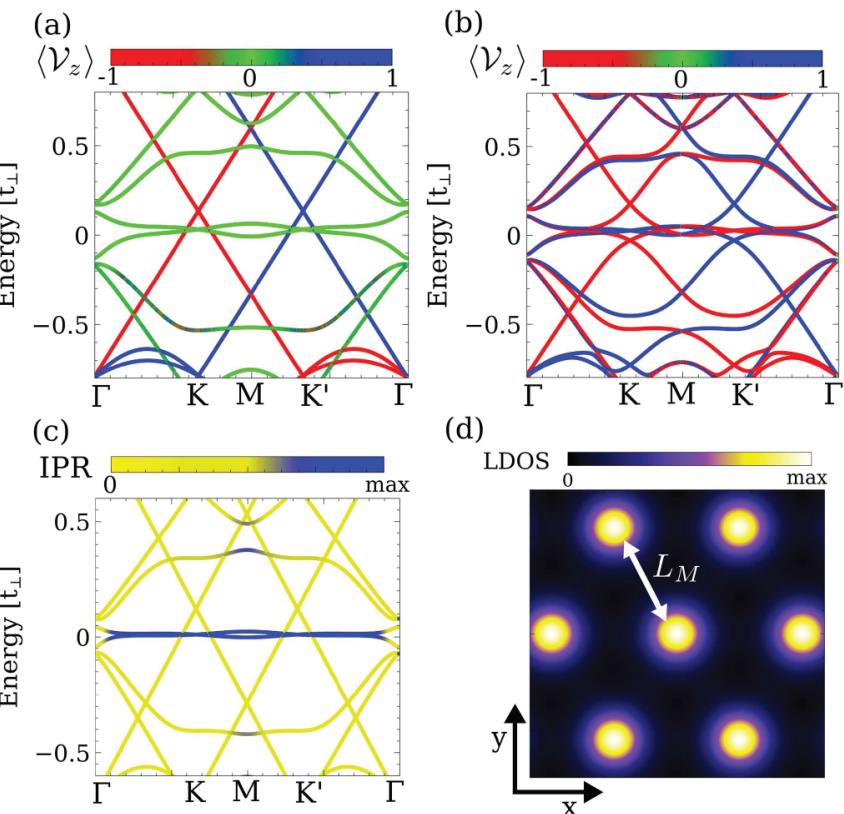

(d)

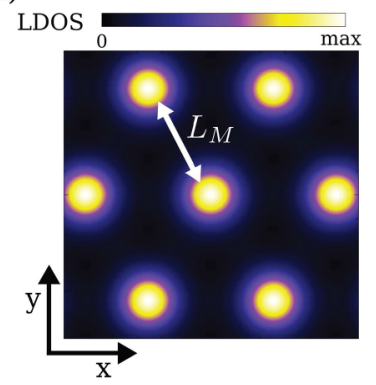

FIG. 3. (a) Band structure of a twisted trilayer at an angle of $1.9^{\circ}$ (a) without and (b) with interlayer bias, showing dispersion analogous to those found by first principles in Fig. 2. (c) Band structure at a lower angle of $\theta=1.6^{\circ}$, showing a flattening of the bands. (d) Local density of states at the Fermi energy, showing a triangular supercell pattern. Note that part of the $k$ path is different in comparison to Fig. 2. Calculations were performed with tight binding.

show two crossings above and below the nearly flat bands, analogously to the first-principles results. It is also observed that the nearly flat bands are slightly modified by the interlayer bias. As we will show below, such effect becomes stronger for even smaller angles.

The last interesting point is related to the localization of the different states in the supercell. This can be characterized by means of the inverse participation ratio (IPR), defined as $\sum_{i}\left|\Psi_{k}(i)\right|^{4}$. Large values of the IPR correspond to states localized in the moiré supercell, whereas small values correspond to states delocalized in the moiré supercell. Focusing now on a structure with a $\theta=1.6^{\circ}$ twisting angle, it is clearly observed that the nearly flat bands show a substantially higher degree of localization than the dispersive bands [Fig. 3(c)]. In particular, the flat band states are associated with an emergent triangular lattice in the supercell, as shown in the local density of states (LDOS) of Fig. 3(d). In the following we will see how these flat band states can be electrically controlled and how the bias creates bulk valley currents associated with the valley splittings.

\section{BAND FLATTENING AND VALLEY CURRENTS BY AN INTERLAYER BIAS}

We now move on to systematically analyze the effect of an interlayer bias in the twisted graphene trilayer and in particular its effect on the low-energy density of states. Electric biases in twisted graphene multilayers are known to give rise 


\section{(a)}

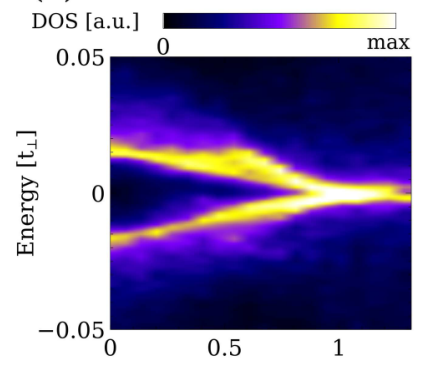

(b)

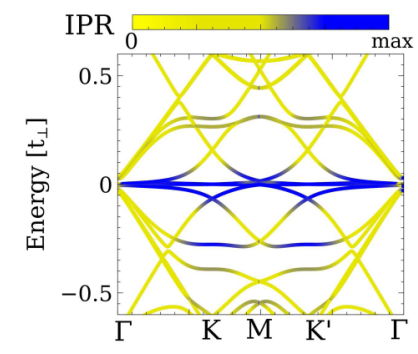

(c)

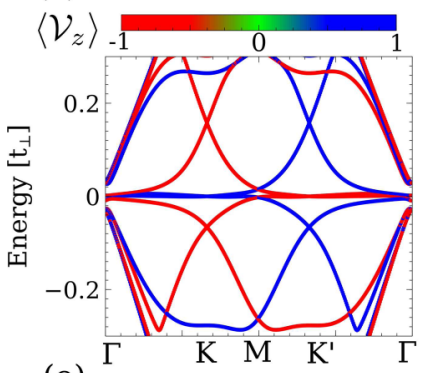

(e)

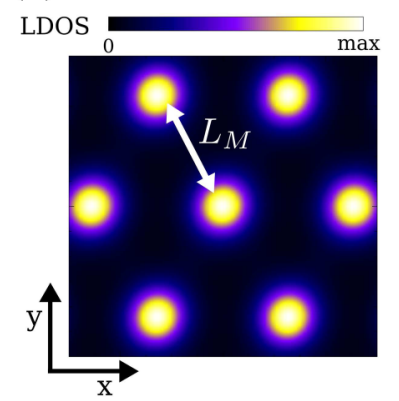

(d)

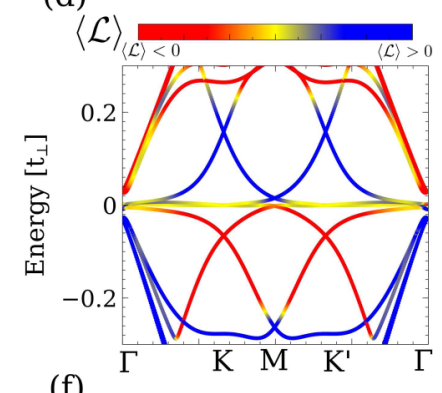

(f)

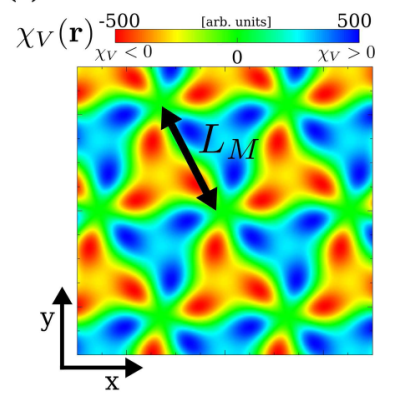

FIG. 4. (a) Evolution of the density of states as a function of the interlayer bias for $\theta=1.6^{\circ}$. (b) Band structure at a finite electric field depicting the localization of each state. (c) Valley polarization of the low-energy states and (d) their layer polarization. (e) Spatial localization of the nearly flat bands. (f) Local valley flux at one electron per unit cell. The spatial maps in (e) and (f) are summed over the three layers. Calculations were performed with tight binding.

to valley Hall currents $[5,6,21,57-60]$ and represent an effective knob to control the low-energy electronic structure [18]. In the following we focus on the structure at $\theta=1.6^{\circ}$. As shown in Fig. 4(a), the application of an interlayer bias merges the original two Van Hove singularities of zero bias in a single one, dramatically enhancing the low-energy density of states. Interestingly, this merging of Van Hove singularities is similar to the evolution with the twist angle in twisted bilayers $[10,42,61]$, with the key difference that in the present case the merging is electrical, turning the process highly controllable in situ.

We now focus on the flattest regime with finite bias, when the two Van Hove singularities get merged. Figures 4(b)-4(d) show the band structure of that regime. It is observed that almost perfectly flat bands are present at the Fermi energy [Figs. 4(b)-4(d)], accounting for the Van Hove singularity at charge neutrality [Fig. 4(a)]. It is also observed that the states remain highly localized in the unit cell as highlighted by the
IPR, whereas at higher energies the dispersive bands are delocalized in the moiré unit cell [Fig. 4(b)]. It is also observed that the bands show perfect valley polarization [Fig. 4(c)], so the interlayer bias creates intervalley scattering, in agreement with continuum models. Given that the interlayer bias breaks the symmetry between the top and bottom layers, it is interesting to look at the layer polarization of the states $\langle\mathcal{L}\rangle$, defined by the layer polarization operator $\mathcal{L}=\sum_{i, s} z_{i} c_{i, s}^{\dagger} c_{i, s}$, with $z_{i}$ the $z$ component of the site $i$. As shown in Fig. 4(d), the Dirac cones at the $K$ and $K^{\prime}$ points above and below the flat bands have a slightly opposite layer polarization. However, such layer polarization is reversed at the $\Gamma$ point [Fig. 4(d)], highlighting that the states remain highly entangled between all the layers.

We now explore how the band flattening is accompanied by the emergence of bulk valley currents. The emergence of valley currents associated with interlayer biases is a well known effect in aligned graphene bilayers [57-60] and tinyangle twisted bilayers $[5,6,21]$. Such valley currents arise due to the emergence of a nonzero local valley Chern number [62] whose quantization is associated with the emergent valley conservation [63]. In twisted systems, the local Chern number is expected to change from region to region [6] due to the locally modulated Hamiltonian. To address this local topological property, in the following we compute the local valley flux by means of the Berry flux density $\chi_{V}$. The real-space valley flux $\chi_{V}$ defines the valley Chern number as $C_{V}=C_{K}-C_{K^{\prime}}=$ $\int \chi_{V}(\mathbf{r}, \omega) d^{2} \mathbf{r} d \omega$. The real-space valley flux $\chi_{V}$ can be computed as $[22,56,64]$

$$
\chi_{V}(\mathbf{r}, \omega)=\int \frac{d^{2} \mathbf{k}}{(2 \pi)^{2}} \frac{\epsilon_{\alpha \beta}}{2}\left\langle\mathbf{r}\left|G_{V}\left(\partial_{k_{\alpha}} G_{V}^{-1}\right)\left(\partial_{k_{\beta}} G_{V}\right)\right| \mathbf{r}\right\rangle,
$$

where $\epsilon_{\alpha \beta}$ denotes the Levi-Cività tensor, $G_{V}$ the valley Green's function $G_{V}=\left[\omega-\mathcal{H}_{\mathbf{k}}+i 0^{+}\right]^{-1} \mathcal{V}_{z}, \mathcal{H}_{k}$ the Bloch Hamiltonian, and $\mathcal{V}_{z}$ the valley polarization operator of Eq. (B1).

Figure 4(f) shows the spatial profile of the valley flux density at the Fermi energy $\chi_{V}(\mathbf{r}) \equiv \chi_{V}\left(\mathbf{r}, \omega=\epsilon_{F}\right)$ for a chemical potential with one hole per unit cell. It can be observed clearly that sizable valley currents appear in regions in the complementary regions to the low-energy states, similarly to other moiré systems [22,56]. Since the emergence of such currents relies on valley conservation, terms in the system creating intervalley mixing are expected to substantially impact them [6,65-67], as we address in the next section.

\section{IMPACT OF CHEMICAL DOPING}

In this section we address the modification of the electronic structure under both electrical and chemical doping. We start with the study of the electric doping from first principles and then move on to consider its effect in the low-energy effective model.

We first address the impact of doping from first principles. First-principles DFT calculations are employed to study the modification of the band diagram of TTG upon doping with both an extra charge and one of the layers with foreign species. Uniform doping of the TTG is realized by introducing an extra electron which is compensated with an equally uniform background charge of opposite sign. This can be realized 


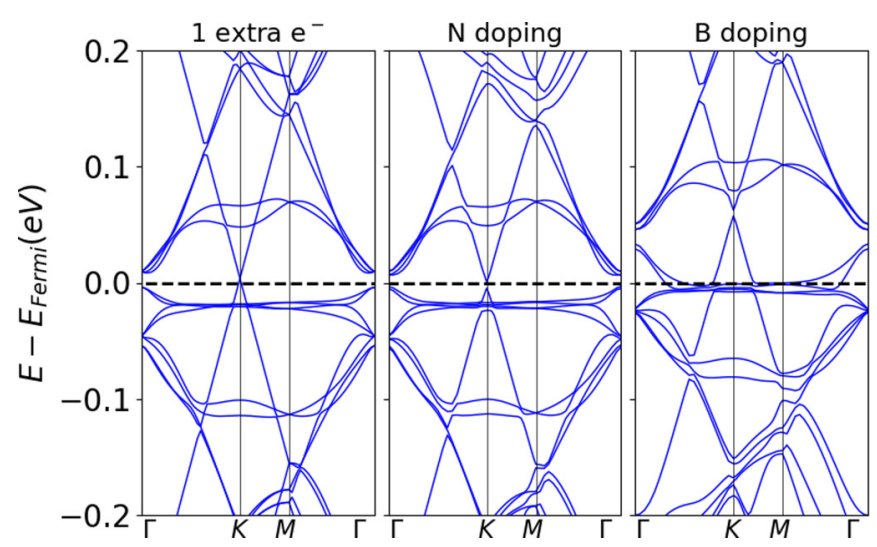

FIG. 5. Electronic band diagrams of three doped TTG configurations. The left panel shows electrostatic doping with one extra electron. The middle panel shows that doping with a $\mathrm{N}$ atom causes a similar effect but removes the Dirac point and converts TTG in a tiny-gap semiconductor. The right panel shows that doping with a $\mathrm{B}$ atom empties some bands at the Fermi level including the highly dispersive bands. Calculations were performed with DFT.

experimentally via a field effect. Figure 5 shows that the extra electron fill the flat states and align the Dirac point with the Fermi energy. Small modifications in the electronic band structure as a result of new electrostatic contributions is observed, similarly to other twisted multilayer systems [68-71].

A similar effect can be induced with chemical doping, namely, adding one $\mathrm{N}$ atom in substitution of a $\mathrm{C}$ atom. The effects of chemical substitution have been extensively studied in graphene [72-81], including twisted bilayers [53,82]. Figure 5 shows the effect on the bands structure of one $\mathrm{N}$ atom in one of the surface layers. The extra charge supplied by the $\mathrm{N}$ atom shifts the chemical potential, increasing the filling of the flat bands. A major difference with respect to the electrostatic doping is the opening of a meV large band gap as a result of the symmetry breaking imposed by the impurity which also induces a mixing of the linear states with the less dispersive states. These new anticrossings are due to the intervalley scattering created by the chemical impurity and they do not appear in the case of electrostatic doping. A similar hybridization of electronic states is also observed when one electron is removed by means of doping with a B atom, as shown in Fig. 5. The chemical potential shift occurs in the opposite direction and the filling of flat band states decreases.

We now consider the effect of a chemical impurity in the tight-binding model. We model the addition of a chemical impurity by adding to the Hamiltonian $\mathcal{H}_{D}=w \sum_{s} c_{i, s}^{\dagger} c_{i, s}$, where $i$ is the site that has been chemically replaced. We take $w=-2 t$, which is the typical energy scale expected for a $N$ dopant that yields results comparable to the firstprinciples calculations. For the sake of concreteness we focus on the case with interlayer bias, so our full Hamiltonian is $\mathcal{H}=\mathcal{H}_{0}+\mathcal{H}_{V}+\mathcal{H}_{D}$. With the previous Hamiltonian, we now compute the electronic band structure and project each eigenstate onto the valley operator. The result is shown in Fig. 6(a), where we see that small anticrossings appear as in the first-principles calculations. The valley projection clearly shows that such anticrossings are associated with intervalley (a)

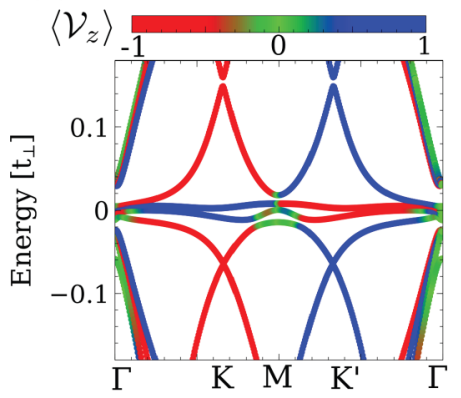

(b)

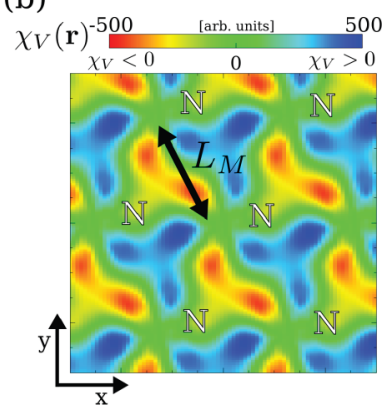

FIG. 6. (a) Band structure in the presence of a $\mathrm{N}$ impurity and (b) valley current at charge neutrality. It can be observed that, although the presence of a dopant creates a nonzero intervalley scattering, the low-energy bands remain relatively flat. The emergence of intervalley scattering can be seen in the depletion of the bulk valley currents in (b). The valley flux in (b) is summed over the three layers. Calculations were performed with tight binding.

mixing. The effect of the impurity in terms of intervalley mixing can also be readily seen in the bulk valley currents. In particular, the existence of the impurity is expected to strongly perturb the original valley fluxes in the unit cell, depleting the local value of the valley Chern number. This is verified in Fig. 6(b), where we observe that the original valley fluxes are impacted by the presence of the impurity.

Interestingly, despite the effect in terms of intervalley mixing, the low-energy bands remain relatively flat, retaining their associated large density of states. This suggests that correlated states can still appear in chemically doped twisted trilayers. It is worth emphasizing that despite this large density of states, correlated phases relying on valley coherent states will be strongly suppressed due to the impurity-induced intervalley mixing. In particular, valley ferromagnet states and valley triplet superconducting states will be depleted due to chemical dopants. Nevertheless, conventional spin singlet and valley singlet states are not affected by the presence of intervalley scattering. Motivated by this, in the next section we address the emergence of spin/valley singlet superconductivity and show how the superfluid density impacts the high-energy dispersive states.

\section{SUPERCONDUCTING STATE}

The large density of states close to charge neutrality suggests that the twisted graphene trilayer can have superconducting instabilities, similarly to twisted bilayers and tetralayers. As shown above, with both electrostatic and chemical doping the system shows a divergent density of state close to charge neutrality. For the sake of concreteness we will now focus on the electrostatically doped system, yet we have verified that our results remain qualitatively unchanged with chemical doping.

An emergent superconducting state is associated with Fermi surface instability, yet its effect can give rise to secondorder perturbations above the Fermi energy. In order to understand the potential impact at high energies, we first briefly analyze the structure of the low-energy states. This can be done by comparing the spatial distribution of the 


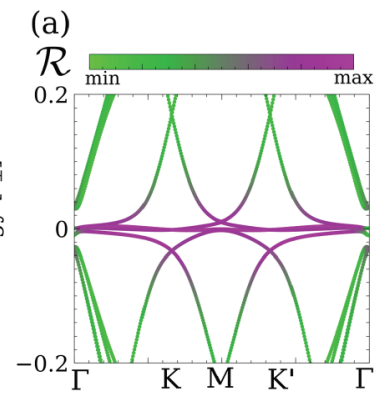

(c)

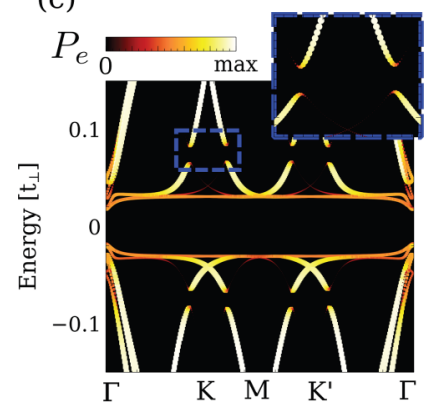

(b)

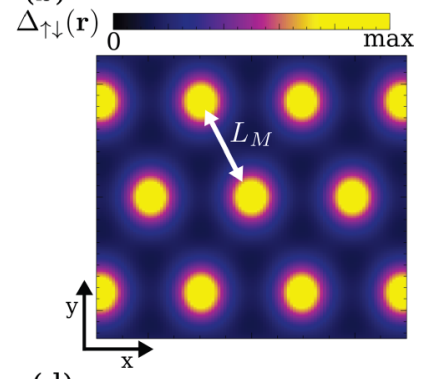

(d)

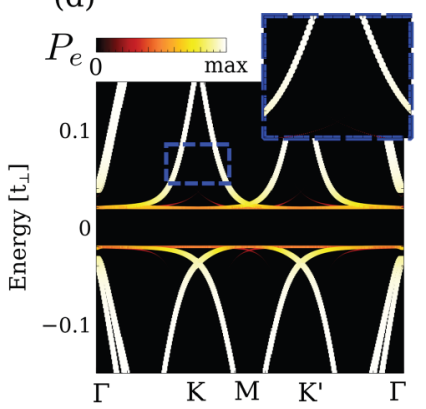

FIG. 7. (a) Normal state band structure in the absence of interactions, with the color denoting the projection of the states on the density of states at the Fermi energy. (b) Upon introducing interactions, a nonzero superfluid density appears. (c) Electronic dispersion associated with the self-consistent solution of (b), where we have projected the eigenstates over the electron sector. (d) Electronic dispersion projected in the electron sector for a uniform superfluid density, highlighting that the high-energy anticrossings stem from the nonuniform pairing. Calculations were performed with tight binding.

low-energy states with respect to the Fermi surface states. In particular, we define the projection over the Fermi surface states as $\mathcal{R}=\int d^{2} \mathbf{r}|\Psi(\mathbf{r})|^{2} \rho_{F}(\mathbf{r})$, with $\rho_{F}(\mathbf{r})$ the local density at the Fermi energy $\rho_{F}(\mathbf{r}) \sim \int\left\langle\mathbf{r}\left|\delta\left(E_{F}-H\right)\right| \mathbf{r}\right\rangle$. The quantity $\mathcal{R}$ allows us to qualitatively distinguish which states are localized in the same region at the Fermi surface states.

By computing the Fermi surface state projector $\mathcal{R}$, it observed that the states of the flat band are localized in similar regions. In contrast, departing from the Fermi energy, the states start to delocalize to other regions of the unit cell [Fig. 7(a)]. This highlights the different orbital nature of the nearly flat bands (in purple) and low-energy dispersive bands (in green). Importantly, and in stark contrast to twisted graphene bilayers, the flat bands of this system are not decoupled from the dispersive states, suggesting that the superconducting states of this system will have a genuine multiorbital nature.

Given the unavoidable entanglement between the flat and dispersive bands, an effective model description of this twisted trilayer cannot be easily performed. Therefore, in the following we study the emergent superconducting state by exploiting the full atomistic model, including all the bands in our calculation. A variety of mechanisms have been suggested to give rise to attractive interactions in these systems, including phonon [83-85], Coulomb [86], and magnon fluctuations $[87,88]$. For the sake of concreteness, we focus on effective local attractive interactions, as employed in other twisted graphene systems [83,84,89-94]

$$
\mathcal{H}_{I}=-\sum_{i} g c_{i, \uparrow}^{\dagger} c_{i, \uparrow} c_{i, \downarrow}^{\dagger} c_{i, \downarrow}
$$

that we solve at the mean field level $\mathcal{H}_{I}^{M F}=$ $-g \sum_{i}\left\langle c_{i, \uparrow}^{\dagger} c_{i, \downarrow}^{\dagger}\right\rangle c_{i, \uparrow} c_{i, \downarrow}+$ H.c., where $\left\langle c_{i, \uparrow}^{\dagger} c_{i, \downarrow}^{\dagger}\right\rangle$ is computed self-consistently for the full Hamiltonian $\mathcal{H}=\mathcal{H}_{0}+\mathcal{H}_{V}+$ $\mathcal{H}_{I}^{M F}$, which we solve with the Bogoliubov-de Gennes (BdG) formalism. The local attractive interaction $g$ will give rise to a net superfluid density, with a moiré momentum structure of $s$-wave symmetry. In the following we set $g=t$ and we verify that our results remain qualitatively similar with smaller interaction strengths. We note that although interactions are local, that would lead to a nontrivial multiorbital structure in the moiré orbital space.

By solving the previous self-consistent problem, we find that the superfluid density is nonuniform in the moire unit cell [Fig. 7(b)], stemming from the nonuniformity of the low-energy states. By projecting the $\mathrm{BdG}$ eigenstates in the electron sector via the electron projector $P_{e}$, we observe that the net nonuniform superfluid density $\Delta_{\uparrow \downarrow}\left(\mathbf{r}_{i}\right) \sim\left\langle c_{i, \uparrow}^{\dagger} c_{i, \downarrow}^{\dagger}\right\rangle$ gives rise to a full gap in the Brillouin zone in the superconducting state. This phenomenology is similar to the one found in twisted graphene bilayers. More interestingly, besides the gap opening at the chemical potential, anticrossings appear in high-energy bands when the self-consistent pairing is included [Fig. 7(c)]. It is worth emphasizing that, in the presence of a uniform pairing artificially imposed, the high-energy anticrossings disappear, leading only to the gap opening at charge neutrality [Fig. 7(d)]. The emergence of gap openings away from charge neutrality is associated with the intrinsically multiorbital nature of the superconducting state and stems from the nonunitarity of the superconducting matrix in orbital subspace $[95,96]$. Interestingly, this shows that signatures of the superconducting state can be obtained by analyzing the system away from the chemical potential and could provide powerful spectroscopic signatures [97,98] of the superconducting state.

\section{CONCLUSION}

By combining first-principles calculation and low-energy effective models, we have shown that twisted graphene trilayers realize tunable electronic systems. In particular, it was shown that nearly perfect flat bands can be electrically controlled, which coexist with highly dispersive states. Interestingly, such electric flattening of the bands is accompanied by the emergence of bulk valley currents. We have found from both first-principles and low-energy calculations that chemical doping does not destroy the flat bands, yet it substantially impacts the bulk valley currents. This suggests that chemical doping of twisted graphene trilayers could provide an intrinsic way of providing the necessary electronic doping required for the emergence of a superconducting state. We finally demonstrated that an emergent superconducting state would give rise to spectroscopic changes in the high-energy bands, associated with the nonuniform superfluid density. Our results highlight the rich physics of twisted graphene trilayers and provide a starting point to explore the interplay between flat bands, correlation, and dispersive states in twisted graphene multilayers. 


\section{ACKNOWLEDGMENTS}

Los Alamos National Laboratory is managed by Triad National Security, LLC, for the National Nuclear Security Administration of the U.S. Department of Energy under Contract No. 89233218CNA000001. This work was supported by the U.S. DOE Office of Basic Energy Sciences Program (E3B5). A.L.-B. acknowledges the computing resources provided on Bebop, the high-performance computing clusters operated by the Laboratory Computing Resource Center at Argonne National Laboratory. J.L.L. thanks T. Wolf, G. Blatter, M. Sigrist, O. Zilberberg, W. Chen, T. Neupert, A. Ramires, and T. Heikkilä for fruitful discussions. J.L.L. acknowledges the computational resources provided by the Aalto ScienceIT project. J.L.L is grateful for financial support from the Academy of Finland Research Fellowship under Projects No. 331342 and No. 336243.

\section{APPENDIX A: FIRST-PRINCIPLES CALCULATIONS}

A description of the coupling between the graphene layers was conducted through self-consistent calculations with the SIESTA code [99] within a localized orbital basis set scheme. Paramagnetic calculations were conducted using a double- $\zeta$ basis set and the local density approximation approach [100] for the exchange-correlation functional was used. Atomic positions of systems formed by over 5514 atoms were fully relaxed with a force tolerance of $0.02 \mathrm{eV} / \AA$. The integration over the Brillouin zone was performed using a Monkhorst sampling at the $\Gamma$ point. The radial extension of the orbitals had a finite range with a kinetic energy cutoff of $50 \mathrm{meV}$. A vertical separation of $35 \AA$ in the simulation box prevents virtual periodic parallel layers from interacting.

\section{APPENDIX B: VALLEY OPERATOR}

The valley is an emergent quantum number in graphene; as a result, it is not apparent how a valley operator can be described in a real-space tight-binding basis. A simple procedure to define the valley expectation value in a tight-binding model is by noting that for the $z$ component of the valley, we are looking for an operator with eigenvalue +1 for states in one valley and -1 for states in another valley. This would be accomplished by a Hamiltonian that realizes a valley-dependent chemical potential. Let us first focus on a honeycomb lattice and take the real-space operator in a honeycomb lattice $[21,52,55]$

$$
\mathcal{V}_{z}=\frac{i}{3 \sqrt{3}} \sum_{\langle i, j\rangle, s} \eta_{i j} \sigma_{z}^{i j} c_{i, s}^{\dagger} c_{j, s},
$$

where $\langle\langle i, j\rangle\rangle$ denotes next-nearest-neighbor sites, $\eta_{i j}= \pm 1$ for clockwise or counterclockwise hopping, and $\sigma_{z}^{i j}$ is a Pauli matrix associated with the sublattice degree of freedom. The previous operator is diagonal in the sublattice and is proportional to the identity matrix in reciprocal space $\left(\begin{array}{cc}f(\mathbf{k}) & 0 \\ 0 & f(\mathbf{k})\end{array}\right)$, with $f(\mathbf{k})$ the Fourier transform of the real-space hopping. It is easily shown that $f(\mathbf{k}) \approx+1$ close to the $K$ point and $f(\mathbf{k}) \approx$ -1 close the $K^{\prime}$ point, and as a result such next-nearestneighbor hopping allows us to compute the expectation value of the valley for a specific state. In particular, by taking the (a)

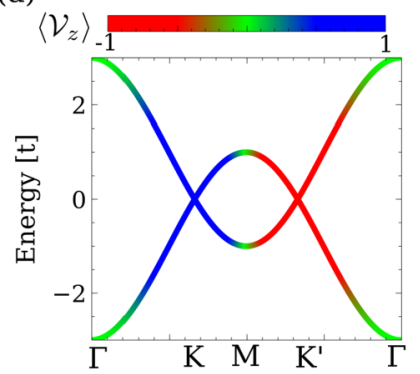

(c)

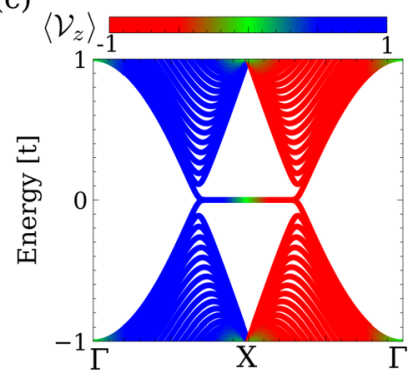

(e)

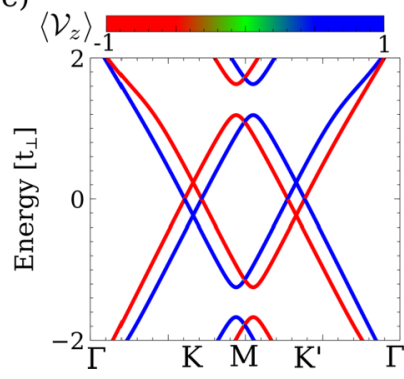

(b)

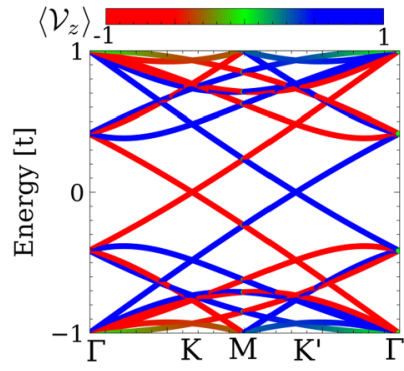

(d)

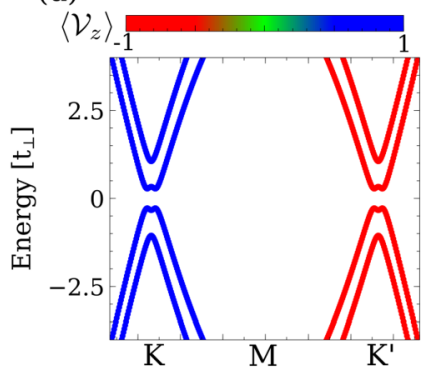

(f)

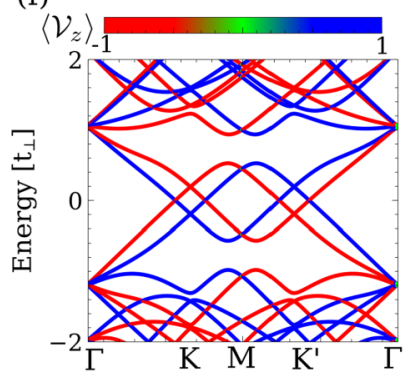

FIG. 8. Valley expectation value for the minimal unit cell (a) of a honeycomb lattice and (b) in an $8 \times 8$ supercell. The valley operator is projected on (c) the states of a graphene zigzag ribbon and (d) a biased aligned Bernal-stacked graphene bilayer. The projection of the valley operator for a biased twisted graphene bilayer is shown for rotation angles of (e) $10^{\circ}$ and (f) $5^{\circ}$. We set $V=0.05 t$ in (d)-(f).

expectation value $\left\langle\mathcal{V}_{z}\right\rangle=\left\langle\Psi\left|\mathcal{V}_{z}\right| \Psi\right\rangle$, we will obtain $\left\langle\mathcal{V}_{z}\right\rangle \approx 1$ if $\Psi$ is a state belonging to valley $K$ and $\left\langle\mathcal{V}_{z}\right\rangle \approx-1$ if $\Psi$ is a state belonging to valley $K^{\prime}$. This can be clearly seen in Fig. 8(a), where we show the valley expectation value for the states of the honeycomb lattice, showing that the states around valley $K$ have eigenvalue +1 and those around $K^{\prime}$ have eigenvalue $-K^{\prime}$.

The valley operator allows us to easily track the valley flavor of the electronic states in various situations. Let us now show some of them for the sake of clarity. The simplest case consists of the electronic structure of a supercell of a honeycomb lattice. In particular, we show in Fig. 8(b) the band structure for an $8 \times 8$ supercell, indicating that the valley operator allows following the original valley flavor of the states in the folded band structure. It is worth noting that such an operator can be defined in a graphene structure, without requiring two-dimensional periodicity, for example, for graphene nanoribbons. In particular, we show in Fig. 8(c) the band structure of a zigzag graphene nanoribbon, demonstrating that the valley operator correctly identifies the valley flavor of each state. 
(a)
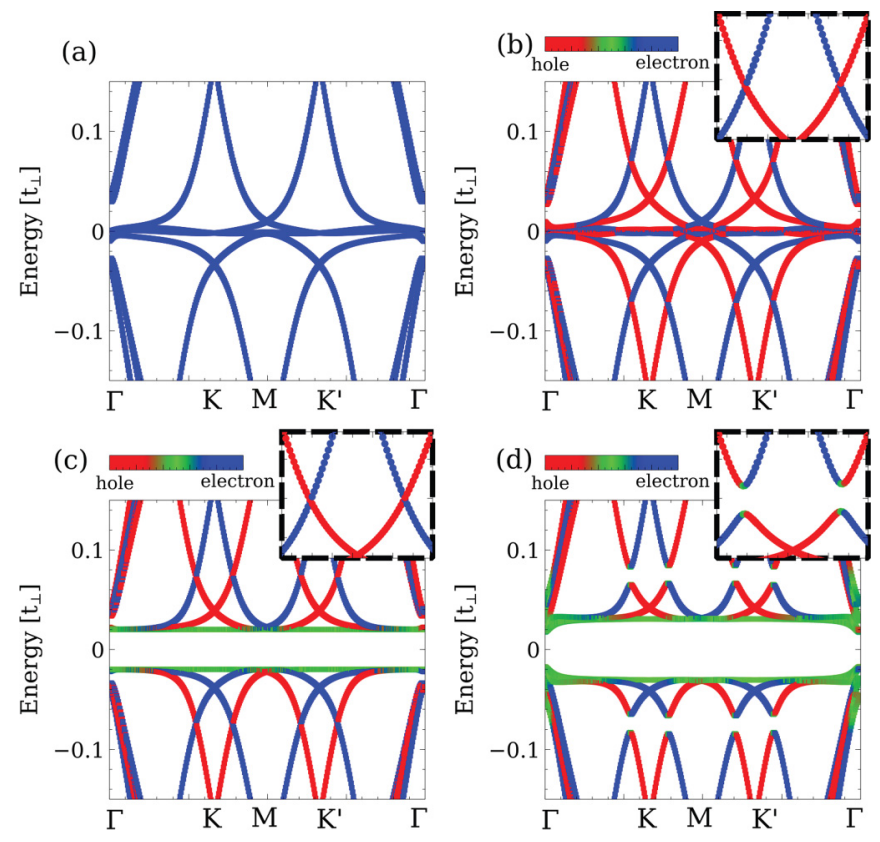

FIG. 9. Band structure of the biased twisted trilayer in the normal state, showing (a) only the electron sector and (b) both the BdG electron and hole sectors. (c) When a nonzero superfluid weight is included, anticrossings in the high-energy bands do not appear for a uniform superfluid matrix $\Delta \sim \mathcal{I}$. (d) In contrast, anticrossings at high energies emerge for a self-consistent nonuniform superfluid weight due to the nonzero matrix element $\Gamma=\left\langle\Psi_{n, \mathbf{k}}|\hat{\Delta}| \Psi_{l,-\mathbf{k}}\right\rangle \neq 0$.

The valley operator can be easily extended to graphene multilayers. In particular, by defining the valley operator of layer $\alpha$ as $\mathcal{V}_{z}^{\alpha}$, the total valley operator is defined as $\mathcal{V}_{z}=$ $\sum_{\alpha} \mathcal{V}_{z}^{\alpha}$. Let us first illustrate this with a simple graphene multilayer, an electrically biased Bernal-stacked graphene bilayer. In this situation shown in Fig. 8(d), we again observe that the multilayer valley operator correctly identifies the states belonging to the different valleys. This very same idea can be used for twisted graphene multilayers. In particular, in Figs. 8(e) and 8(f) we show that in a biased twisted graphene bilayer at angles of $10^{\circ}$ [Fig. 8(e)] and $5^{\circ}$ [Fig. 8(f)], the valley operator correctly identifies the microscopic valley of each state.

\section{APPENDIX C: ORIGIN OF HIGH-ENERGY ANTICROSSINGS IN THE SUPERCONDUCTING STATE}

In the following, we elaborate on the origin of the highenergy anticrossings in the superconducting state. Let us start with the band structure of the biased twisted trilayer graphene, as shown in Fig. 9(a). By extending the spectra in a Bogoliubov-de Gennes formalism, hole replicas of the original states appear. This is shown in Fig. 9(b) for $\Delta=0$, where the blue bands denote electronlike bands and red bands denote the hole replicas. It is worth noting that in this situation, electronlike and holelike bands above the chemical potential cross and therefore a nonzero superfluid weight can potentially lead to anticrossings, as such terms couple electron and hole sectors. Let us now turn to a spatially uniform superfluid weight, as shown in Fig. 9(c). In this situation, it is observed that for the electronlike and holelike bands crossing above the chemical potential, no anticrossings appears. The coupling between the electron and hole states above the chemical potential is proportional to the overlap of the single-particle wave function $\Psi_{n, \mathbf{k}}$ with the superconducting matrix $\hat{\Delta}$ and takes the form $\Gamma=\left\langle\Psi_{n, \mathbf{k}}|\hat{\Delta}| \Psi_{l,-\mathbf{k}}\right\rangle$, with $n \neq l$. For uniform superfluid density $\hat{\Delta} \sim \mathcal{I}$, with $\mathcal{I}$ the identity matrix, we have $\Gamma=0$ from the orthogonality of the wave functions and therefore no anticrossings appear in the high-energy bands of Fig. 9(c). In stark contrast, when the superfluid weight is nonuniform in space we have $\hat{\Delta} \not \subset \mathcal{I}$ and we generically have $\Gamma=\left\langle\Psi_{n, \mathbf{k}}|\hat{\Delta}| \Psi_{l,-\mathbf{k}}\right\rangle \neq 0$, leading to an effective anticrossing between the states. As a result, the appearance of anticrossing in the high-energy bands is a direct consequence of the nonuniform superfluid weight. We finally note that this argument relies on the original time-reversal symmetry of the twisted trilayer graphene Hamiltonian.
[1] Y. Cao, V. Fatemi, S. Fang, K. Watanabe, T. Taniguchi, E. Kaxiras, and P. Jarillo-Herrero, Nature (London) 556, 43 (2018).

[2] M. Yankowitz, S. Chen, H. Polshyn, Y. Zhang, K. Watanabe, T. Taniguchi, D. Graf, A. F. Young, and C. R. Dean, Science 363, 1059 (2019).

[3] K. Liu, L. Zhang, T. Cao, C. Jin, D. Qiu, Q. Zhou, A. Zettl, P. Yang, S. G. Louie, and F. Wang, Nat. Commun. 5, 4966 (2014).

[4] X. Lu, P. Stepanov, W. Yang, M. Xie, M. A. Aamir, I. Das, C. Urgell, K. Watanabe, T. Taniguchi, G. Zhang, A. Bachtold, A. H. MacDonald, and D. K. Efetov, Nature (London) 574, 653 (2019).

[5] P. Rickhaus, J. Wallbank, S. Slizovskiy, R. Pisoni, H. Overweg, Y. Lee, M. Eich, M.-H. Liu, K. Watanabe, T. Taniguchi, T. Ihn, and K. Ensslin, Nano Lett. 18, 6725 (2018).

[6] P. San-Jose and E. Prada, Phys. Rev. B 88, 121408(R) (2013).
[7] M. Liao, Z. Wei, L. Du, Q. Wang, J. Tang, H. Yu, F. Wu, J. Zhao, X. Xu, B. Han, K. Liu, P. Gao, T. Polcar, Z. Sun, D. Shi, R. Yang, and G. Zhang, Nat. Commun. 11, 2153 (2020).

[8] Y. Shimazaki, I. Schwartz, K. Watanabe, T. Taniguchi, M. Kroner, and A. Imamoğlu, Nature (London) 580, 472 (2020).

[9] J. M. B. Lopes dos Santos, N. M. R. Peres, and A. H. Castro Neto, Phys. Rev. Lett. 99, 256802 (2007).

[10] E. Suárez Morell, J. D. Correa, P. Vargas, M. Pacheco, and Z. Barticevic, Phys. Rev. B 82, 121407(R) (2010).

[11] M. Serlin, C. L. Tschirhart, H. Polshyn, Y. Zhang, J. Zhu, K. Watanabe, T. Taniguchi, L. Balents, and A. F. Young, Science 367, 900 (2019).

[12] S. J. Ahn, P. Moon, T.-H. Kim, H.-W. Kim, H.-C. Shin, E. H. Kim, H. W. Cha, S.-J. Kahng, P. Kim, M. Koshino, Y.-W. Son, C.-W. Yang, and J. R. Ahn, Science 361, 782 (2018).

[13] P. Moon, M. Koshino, and Y.-W. Son, Phys. Rev. B 99, 165430 (2019). 
[14] G. Yu, Z. Wu, Z. Zhan, M. I. Katsnelson, and S. Yuan, npj Comput. Mater. 5, 122 (2019).

[15] S. Pezzini, V. Miseikis, G. Piccinini, S. Forti, S. Pace, R. Engelke, F. Rossella, K. Watanabe, T. Taniguchi, P. Kim, and C. Coletti, Nano Lett. 20, 3313 (2020).

[16] S. Chen, M. He, Y.-H. Zhang, V. Hsieh, Z. Fei, K. Watanabe, T. Taniguchi, D. H. Cobden, X. Xu, C. R. Dean, and M. Yankowitz, arXiv:2004.11340.

[17] H. Polshyn, J. Zhu, M. A. Kumar, Y. Zhang, F. Yang, C. L. Tschirhart, M. Serlin, K. Watanabe, T. Taniguchi, A. H. MacDonald, and A. F. Young, arXiv:2004.11353.

[18] E. V. Castro, K. S. Novoselov, S. V. Morozov, N. M. R. Peres, J. M. B. Lopes dos Santos, J. Nilsson, F. Guinea, A. K. Geim, and A. H. Castro Neto, Phys. Rev. Lett. 99, 216802 (2007).

[19] X. Liu, Z. Hao, E. Khalaf, J. Y. Lee, K. Watanabe, T. Taniguchi, A. Vishwanath, and P. Kim, Nature (London) 583, 221 (2020).

[20] C. Shen, Y. Chu, Q. Wu, N. Li, S. Wang, Y. Zhao, J. Tang, J. Liu, J. Tian, K. Watanabe, T. Taniguchi, R. Yang, Z. Y. Meng, D. Shi, O. V. Yazyev, and G. Zhang, Nat. Phys. 16, 520 (2020).

[21] A. Ramires and J. L. Lado, Phys. Rev. Lett. 121, 146801 (2018).

[22] T. M. R. Wolf, J. L. Lado, G. Blatter, and O. Zilberberg, Phys. Rev. Lett. 123, 096802 (2019).

[23] A. Abouelkomsan, Z. Liu, and E. J. Bergholtz, Phys. Rev. Lett. 124, 106803 (2020).

[24] Z. Liu, A. Abouelkomsan, and E. J. Bergholtz, arXiv:2004.09522.

[25] C. Repellin and T. Senthil, Phys. Rev. Research 2, 023238 (2020).

[26] P. J. Ledwith, G. Tarnopolsky, E. Khalaf, and A. Vishwanath, Phys. Rev. Research 2, 023237 (2020).

[27] S. Carr, D. Massatt, S. B. Torrisi, P. Cazeaux, M. Luskin, and E. Kaxiras, Phys. Rev. B 98, 224102 (2018).

[28] X. Lin, D. Liu, and D. Tománek, Phys. Rev. B 98, 195432 (2018).

[29] N. N. T. Nam and M. Koshino, Phys. Rev. B 96, 075311 (2017).

[30] N. Leconte, S. Javvaji, J. An, and J. Jung, arXiv:1910.12805.

[31] I. Brihuega and F. Yndurain, J. Phys. Chem. B 122, 595 (2017).

[32] M. Koshino and Y.-W. Son, Phys. Rev. B 100, 075416 (2019).

[33] M. Angeli, E. Tosatti, and M. Fabrizio, Phys. Rev. X 9, 041010 (2019).

[34] P. Rickhaus, G. Zheng, J. L. Lado, Y. Lee, A. Kurzmann, M. Eich, R. Pisoni, C. Tong, R. Garreis, C. Gold, M. Masseroni, T. Taniguchi, K. Wantanabe, T. Ihn, and K. Ensslin, Nano Lett. 19, 8821 (2019).

[35] H. Ochoa, Phys. Rev. B 100, 155426 (2019).

[36] E. Suárez Morell, M. Pacheco, L. Chico, and L. Brey, Phys. Rev. B 87, 125414 (2013).

[37] X. Li, F. Wu, and A. H. MacDonald, arXiv:1907.12338.

[38] E. Khalaf, A. J. Kruchkov, G. Tarnopolsky, and A. Vishwanath, Phys. Rev. B 100, 085109 (2019).

[39] S. Carr, C. Li, Z. Zhu, E. Kaxiras, S. Sachdev, and A. Kruchkov, Nano Lett. 20, 3030 (2020).

[40] C. Mora, N. Regnault, and B. A. Bernevig, Phys. Rev. Lett. 123, 026402 (2019).

[41] Y. Park, B. Lingam Chittari, and J. Jung, Phys. Rev. B 102, 035411 (2020).
[42] R. Bistritzer and A. H. MacDonald, Proc. Natl. Acad. Sci. USA 108, 12233 (2011).

[43] M. Angeli, D. Mandelli, A. Valli, A. Amaricci, M. Capone, E. Tosatti, and M. Fabrizio, Phys. Rev. B 98, 235137 (2018).

[44] P. Lucignano, D. Alfè, V. Cataudella, D. Ninno, and G. Cantele, Phys. Rev. B 99, 195419 (2019).

[45] G. Cantele, D. Alfè, F. Conte, V. Cataudella, D. Ninno, and P. Lucignano, arXiv:2004.14323.

[46] F. Haddadi, Q. Wu, A. J. Kruchkov, and O. V. Yazyev, Nano Lett. 20, 2410 (2020).

[47] K. Kim, M. Yankowitz, B. Fallahazad, S. Kang, H. C. P. Movva, S. Huang, S. Larentis, C. M. Corbet, T. Taniguchi, K. Watanabe, S. K. Banerjee, B. J. LeRoy, and E. Tutuc, Nano Lett. 16, 1989 (2016).

[48] Y. Su and S.-Z. Lin, Phys. Rev. B 98, 195101 (2018).

[49] L. A. Gonzalez-Arraga, J. L. Lado, F. Guinea, and P. San-Jose, Phys. Rev. Lett. 119, 107201 (2017).

[50] F. J. Culchac, R. B. Capaz, L. Chico, and E. Suarez Morell, Nanoscale 12, 5014 (2020).

[51] E. S. Morell, P. Vargas, P. Häberle, S. A. Hevia, and L. Chico, Phys. Rev. B 91, 035441 (2015).

[52] A. Ramires and J. L. Lado, Phys. Rev. B 99, 245118 (2019).

[53] A. Lopez-Bezanilla and J. L. Lado, Phys. Rev. Mater. 3, 084003 (2019).

[54] J. M. B. Lopes dos Santos, N. M. R. Peres, and A. H. Castro Neto, Phys. Rev. B 86, 155449 (2012).

[55] E. Colomés and M. Franz, Phys. Rev. Lett. 120, 086603 (2018).

[56] A. L. R. Manesco, J. L. Lado, E. V. S. Ribeiro, G. Weber, and D. Rodrigues, Jr., arXiv:2003.05163.

[57] E. McCann, Phys. Rev. B 74, 161403(R) (2006).

[58] I. Martin, Y. M. Blanter, and A. F. Morpurgo, Phys. Rev. Lett. 100, 036804 (2008).

[59] F. Zhang, A. H. MacDonald, and E. J. Mele, Proc. Natl. Acad. Sci. USA 110, 10546 (2013).

[60] J. L. Mañes, F. Guinea, and M. A. H. Vozmediano, Phys. Rev. B 75, 155424 (2007).

[61] P. San-Jose, J. González, and F. Guinea, Phys. Rev. Lett. 108, 216802 (2012).

[62] Y. Ren, Z. Qiao, and Q. Niu, Rep. Prog. Phys. 79, 066501 (2016).

[63] A. H. Castro Neto, F. Guinea, N. M. R. Peres, K. S. Novoselov, and A. K. Geim, Rev. Mod. Phys. 81, 109 (2009).

[64] K.-T. Chen and P. A. Lee, Phys. Rev. B 84, 205137 (2011).

[65] G. Tkachov and M. Hentschel, Phys. Rev. B 86, 205414 (2012).

[66] B. Yan, Q. Han, Z. Jia, J. Niu, T. Cai, D. Yu, and X. Wu, Phys. Rev. B 93, 041407(R) (2016).

[67] J. N. B. Rodrigues, Phys. Rev. B 94, 134201 (2016).

[68] T. Cea, N. R. Walet, and F. Guinea, Phys. Rev. B 100, 205113 (2019).

[69] F. Guinea and N. R. Walet, Proc. Natl. Acad. Sci. USA 115, 13174 (2018).

[70] P. A. Pantaleon, T. Cea, R. Brown, N. R. Walet, and F. Guinea, arXiv:2003.05050.

[71] Z. A. H. Goodwin, V. Vitale, X. Liang, A. A. Mostofi, and J. Lischner, arXiv:2004.14784.

[72] S. J. Brun, V. M. Pereira, and T. G. Pedersen, Phys. Rev. B 93, 245420 (2016).

[73] B. Wang and S. T. Pantelides, Phys. Rev. B 83, 245403 (2011). 
[74] H. Liu, Y. Liu, and D. Zhu, J. Mater. Chem. 21, 3335 (2011).

[75] A. Lopez-Bezanilla, F. Triozon, and S. Roche, Nano Lett. 9 , 2537 (2009).

[76] S. Agnoli and M. Favaro, J. Mater. Chem. A 4, 5002 (2016).

[77] V. Georgakilas, M. Otyepka, A. B. Bourlinos, V. Chandra, N. Kim, K. C. Kemp, P. Hobza, R. Zboril, and K. S. Kim, Chem. Rev. 112, 6156 (2012).

[78] C. Si, W. Duan, Z. Liu, and F. Liu, Phys. Rev. Lett. 109, 226802 (2012).

[79] K. Pi, W. Han, K. M. McCreary, A. G. Swartz, Y. Li, and R. K. Kawakami, Phys. Rev. Lett. 104, 187201 (2010).

[80] A. López-Bezanilla, F. Triozon, S. Latil, X. Blase, and S. Roche, Nano Lett. 9, 940 (2009).

[81] A. Altland, Phys. Rev. Lett. 97, 236802 (2006).

[82] H. Yang, Z.-Q. Gao, and F. Wang, arXiv:1908.09555.

[83] T. J. Peltonen, R. Ojajärvi, and T. T. Heikkilä, Phys. Rev. B 98, 220504(R) (2018).

[84] F. Wu, A. H. MacDonald, and I. Martin, Phys. Rev. Lett. 121, 257001 (2018).

[85] B. Lian, Z. Wang, and B. A. Bernevig, Phys. Rev. Lett. 122, 257002 (2019).

[86] J. González and T. Stauber, Phys. Rev. Lett. 122, 026801 (2019).

[87] C. Xu and L. Balents, Phys. Rev. Lett. 121, 087001 (2018).

[88] C.-C. Liu, L.-D. Zhang, W.-Q. Chen, and F. Yang, Phys. Rev. Lett. 121, 217001 (2018).
[89] E. Zhao and A. Paramekanti, Phys. Rev. Lett. 97, 230404 (2006).

[90] B. Uchoa and A. H. Castro Neto, Phys. Rev. Lett. 98, 146801 (2007).

[91] N. B. Kopnin and E. B. Sonin, Phys. Rev. Lett. 100, 246808 (2008).

[92] A. Julku, T. J. Peltonen, L. Liang, T. T. Heikkilä, and P. Törmä, Phys. Rev. B 101, 060505(R) (2020).

[93] T. T. Heikkilä and T. Hyart, Europhys. News 50, 24 (2019).

[94] X. Hu, T. Hyart, D. I. Pikulin, and E. Rossi, Phys. Rev. Lett. 123, 237002 (2019).

[95] J. L. Lado and M. Sigrist, Phys. Rev. Res. 1, 033107 (2019).

[96] N. Zaki, G. Gu, A. M. Tsvelik, C. Wu, and P. D. Johnson, arXiv:1907.11602.

[97] B. Amorim, Phys. Rev. B 97, 165414 (2018).

[98] S. Lisi, X. Lu, T. Benschop, T. A. de Jong, P. Stepanov, J. R. Duran, F. Margot, I. Cucchi, E. Cappelli, A. Hunter, A. Tamai, V. Kandyba, A. Giampietri, A. Barinov, J. Jobst, V. Stalman, M. Leeuwenhoek, K. Watanabe, T. Taniguchi, L. Rademaker, S. J. van der Molen, M. Allan, D. K. Efetov, and F. Baumberger, arXiv:2002.02289.

[99] J. M. Soler, E. Artacho, J. D. Gale, A. García, J. Junquera, P. Ordejón, and D. Sánchez-Portal, J. Phys.: Condens. Matter 14, 2745 (2002).

[100] W. Kohn and L. J. Sham, Phys. Rev. 140, A1133 (1965). 